\title{
PID Heating and Temperature Control Method Based On Dynamic Assignment
}

\author{
Yu Feng Zhang ${ }^{1, a, *}$, Ming Li ${ }^{1, b}$ and Jing Min Dai ${ }^{2, c}$ \\ ${ }^{1}$ College of New Energy, Bohai University, Jinzhou, China \\ ${ }^{2}$ School of Electrical Engineering and Automation, Harbin Institute of Technology, Harbin, China \\ a zyf81@aliyun.com, b 1140463824@qq.com, c djm@hit.edu.cn \\ *corresponding author
}

Keywords: PID-DTA control, High vacuum, Heating method, Temperature overshoot

\begin{abstract}
In the process of heating for materials, ordinary PID control methods cause the high temperature overshoot, which damages the structure and properties of the sample. In views of the large thermal inertia under the vacuum system, A PID control based on dynamic temperature assignment (PID-DTA) was proposed. According to temperature differences between the current temperature and target temperature, the assignment temperature of PID controller is changed until the heating temperature reaches the set value. The PID-DTA heating method is validated by experiments using the high vacuum heating system. It is proved by experiment that the PID-DTA heating method effectively reduces the temperature overshoot of heating, and shortens the stability time of temperature control.
\end{abstract}

\section{Introduction}

Since the advent of Proportion Integration Differentiation(PID) control theory, PID controller based on the various algorithms has been widely used in many industries ${ }^{[1]}$. Especially in the field of temperature control, $90 \%$ of controller is designed based on the principle of PID ${ }^{[2]}$. For some special applications, there are many improved PID control methods had been adopted ${ }^{[3]}$. These improvements are mainly dependent on the introduction and integration of other control theory such as fuzzy theory ${ }^{[4-6]}$, neural networks ${ }^{[7]}$, Robust theory ${ }^{[8]} \cdot 1980$ s, to meet the complexity of the control object, PID control with capabilities of self-tuning using different algorithms has been proposed $^{[9-11]}$.

In general, emissivity measurement on the high temperature must be performed in the high vacuum environment to protect the blackbody and heating materials ${ }^{[12]}$. In the process of heating for materials, ordinary PID control methods cause the high temperature overshoot, which damages the structure and properties of the test sample ${ }^{[13]}$. In addition, the temperature oscillation caused by high temperature overshoot greatly prolongs the stability time of temperature control. It is main reason causes in the temperature overshoot that the thermal inertia of the vacuum system is relatively large.

Aim to restrain the overshoot of temperature in the process of heating under the vacuum environment, the PID control based on dynamic temperature assignment (PID-DTA)is proposed in this paper.

\section{PID-DTA Heating Control Principle}

PID-DTA heating method, according to temperature differences between the current temperature and target temperature, continuously changes the assignment temperature of PID controller by judging the heating temperature at the present moment, until the heating temperature reaches the set value.

In the process of PID-DTA heating, through the power-regulating role of PID in the temperature controller, the heating power is reduced with the decreases of temperature difference between the current temperature and the target temperature. It is important that multiple and dynamic assignments of temperature promote the regulation parameters of PID to adjust quickly and timely. 
Dynamic change of the target temperature value is the key to improve the adjustment rate of heating power.

When the heating temperature is close to the setting value, the heating power is timely and fast to reduce or stop the heating. Thermal inertia of heating device in the in the vacuum environment is made a full use. The temperature overshoot is avoided effectively by gradually achieving the target value.

The principle of PID-DTA heating method is shown in Figure 1. With the increase of the assignment number $i$, temperature $T$ of the heating material is increased gradually, and difference between the current temperature and target temperature is gradually decreased. Incremental value of dynamic assignment temperature is increasingly close to 0 . The assignment temperature is changed repeatedly until its value is equal to the set value, the assignment of equal.

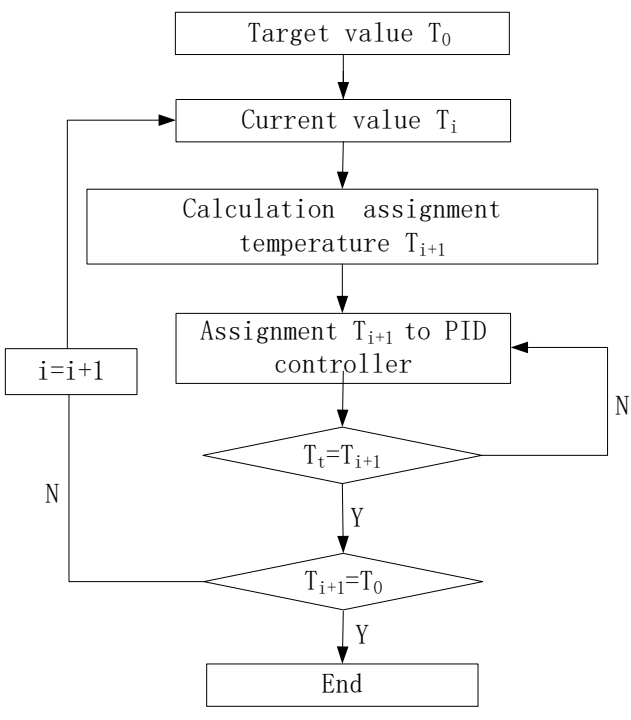

Figure 1 Principle of PID-DTA heating method

The calculated value of assignment temperature $T_{\mathrm{i}+1}$ is determined by target temperature $T_{0}$ and the current temperature $T_{\mathrm{i}}$. which is described by the following formula:

$$
T_{i+1}=T_{i}+\frac{1}{2}\left(T_{0}-T_{i}\right)
$$

\section{Experiment device}

In order to validate the PID-DTA heating method, one part of high vacuum emissivity measurement facility is used for experiments of the heating method. The experimental system consists of the vacuum device, heating apparatus and control cabinet.

\subsection{High Vacuum device}

As shown in Figure 2, the vacuum device mainly consists of a vacuum chamber, a mechanical pump and molecular pump, a series of control valve. The control of vacuum device is carry out by the high vacuum flapper valve and three solenoid valves (pre-pumping valve, front valve and bleeder valve). Through the electrical signal, each of valves was controlled to open or close, and achieve the control of the direction of the airflow in vacuum devices. 


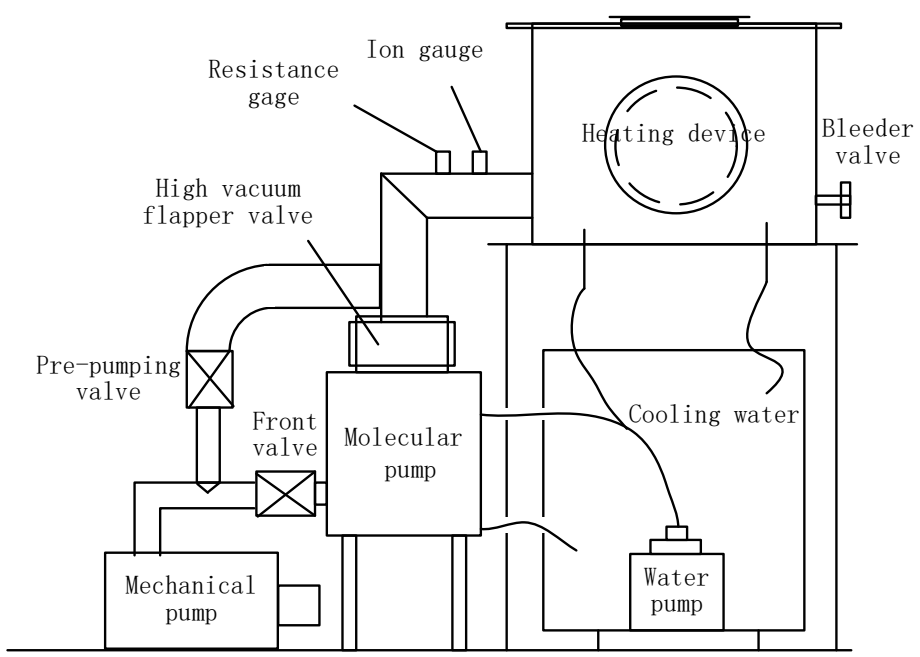

Figure 2 Principle diagram of high vacuum device

\subsection{Heating apparatus}

The apparatus of heating and temperature control is consist of a PID temperature controller, a power regulator, transformer, a heating wire, a heat conducting body, which can be seen in figure 3 .

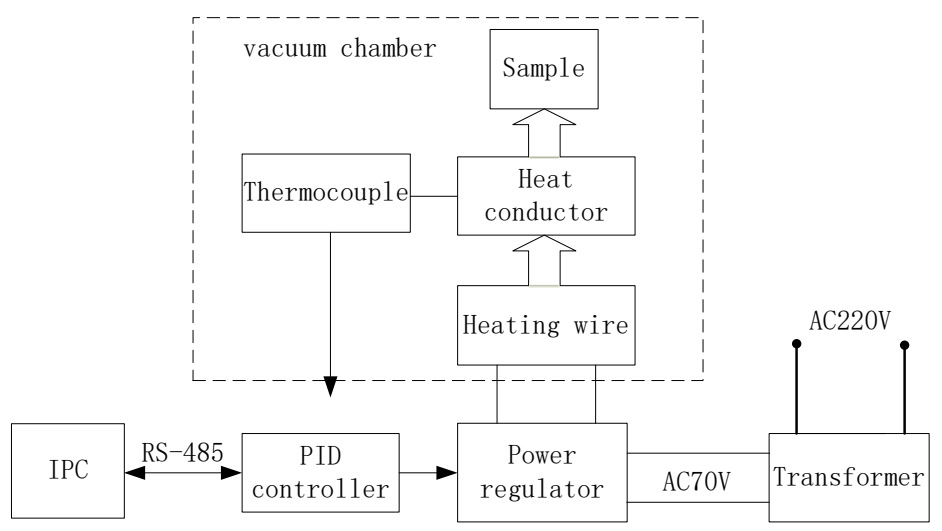

Figure 3 Block diagram of the temperature control

Because the heating device is working in the high vacuum environment (10-3Pa), the higher voltage is easy to cause the ionization discharge of metal electrodes. In the vacuum chamber the heating voltage is changed to $\mathrm{AC} 70 \mathrm{~V}$ through a transformer. According to the feedback temperature signal of thermocouple, temperature controller can timely adjust the heating power to realize temperature control of the heating devices.

\section{Results}

The specimen of coatings used for solar collecting tube was used for the heating experiment. The target value of heating temperature was set to 673K. The temperature of sample with common PID and PID-DTA heating method is record respectively. It is can be seen in Figure 4 that the overshoot temperature and stable time of the common PID method is compared with the method base on the PID-DTA. 


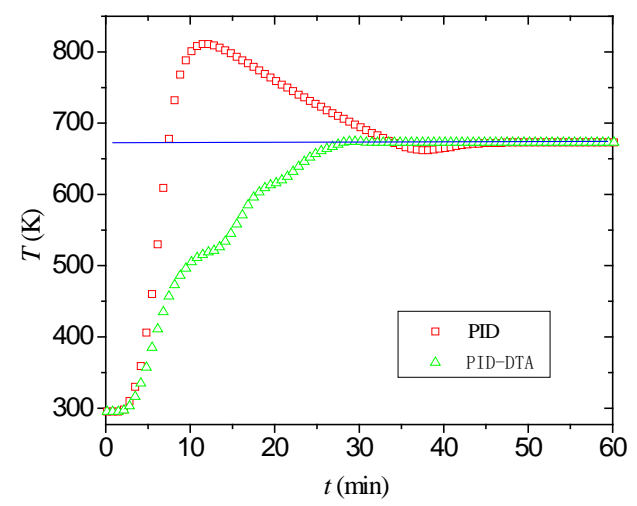

Figure 4 contrast experiment of PID and PID-DTA heating method

In the experiment of heating with the common PID method, the serious overshoot appears. And the highest overshoot temperature has reached to $280 \mathrm{~K}$.

However, the maximum overshoot temperature of PID-DTA heating method is controlled well and its value is within 4K. In addition, the temperature stability time of PID heating method is about 45 minutes, and the PID-DTA is just 35 minutes. Compared with the conventional PID control, the stability time of temperature control was greatly shortened.

\section{Conclusion}

Intelligent PID mode of temperature controller itself can accomplish a good temperature control of heating under atmospheric conditions. In the high vacuum environment, there is only the radiation heat transfer between the sample and the environment. When the setting value of target temperature is changed, self-tuning PID parameters is not able to restrain the overshoot temperature.

Making good use of the thermal inertia of heating devices, PID-DTA heating method is able to change the assignment temperature of PID controller according to temperature differences between the current temperature and target temperature. It is proved by experiment PID-DTA heating method effectively reduces the temperature overshoot of heating, and shorten the stability time of temperature control.

\section{Acknowledgements}

This paper is based upon work supported by the National Science Foundation of China (grant no. 61575029).

\section{References}

[1] Maganioti, A.E., Chrissanthi, H.D., Charalabos, P.C., Andreas, R.D., George, P.N. and Christos, C.N. (2010) Cointegration of Event-Related Potential (ERP) Signals in Experiments with Different Electromagnetic Field (EMF) Conditions. Health, 2, 400-406.

[2] Bootorabi, F., Haapasalo, J., Smith, E., Haapasalo, H. and Parkkila, S. (2011) Carbonic Anhydrase VII-A Potential Prognostic Marker in Gliomas. Health, 3, 6-12.

[1] Ang K.H., Chong G., Li Y.. Pid Control System Analysis, Design, And Technology [J]. IEEE Transactions On Control Systems Technology, 2005, 13(4): 559-566.

[2] Gadoue S.M., Giaouris D., Finch J. W.. MRAS Sensorless Vector Control Of An Induction Motor Using New Sliding-Mode And Fuzzy-Logic Adaptation Mechanisms [J]. IEEE Transactions on Energy Conversion, 2010, 25(2): 394-402.

[3] Radke F., Isermann R.. A Parameter-Adaptive Pid-Controller With Stepwise Parameter Optimization [J]. Automatica, 1987, 23(4): 449-457. 
[4] Fu Y., Chai T.. Neural-Network-Based Nonlinear Adaptive Dynamical Decoupling Control [J]. IEEE Transactions on Neural Networks, 2007, 18(3): 921-925.

[5] Qiao W.Z., Mizumoto M.. PID Type Fuzzy Controller And Parameters Adaptive Method [J]. Fuzzy sets and systems, 1996, 78(1): 23-35.

[6] Woo Z.W., Chung H.Y., Lin J.J.. A PID Type Fuzzy Controller With Self-Tuning Scaling Factors [J]. Fuzzy sets and systems, 2000, 115(2): 321-6.

[7] Peng Y., Wei W., Wang J.. Model Predictive Synchronous Control Of Barrel Temperature For Injection Molding Machine Based on Diagonal Recurrent Neural Networks [J]. Materials and Manufacturing Processes, 2012, 28(1): 24-30.

[8] Soyguder S., Karakose M., Alli H.. Design and Simulation Of Self-Tuning Pid-Type Fuzzy Adaptive Control for An Expert Hvac System [J]. Expert Systems with Applications, 2009, 36(3): 4566-4573.

[9] Fu Y., ChaI T.. Robust Self-Tuning PIDecoupling Control of Uncertain Multivariable Systems [J]. International Journal of Adaptive Control and Signal Processing, 2012, 26(4): 316-32.

[10] Cameron F., Seborg D.. A Self-Tuning Controller with a Pid Structure [J]. International Journal of Control, 1983, 38(2): 401-407.

[11] Gawthrop P.J.. Self-tuning PID Controllers: Algorithms and Implementation [J]. Automatic Control, IEEE Transactions on, 1986, 31(3): 201-209.

[12] Hameury J., Hay B., Filtz J.. Measurement of Total Hemispherical Emissivity Using a Calorimetric Technique [J]. International Journal of Thermophysics, 2007, 28(5): 1607-1620. 